\title{
IncRNA XIST interacts with miR-140 to modulate lung cancer growth by targeting iASPP
}

\author{
YONGJUN TANG, RUOXI HE, JIAN AN, PENGBO DENG, LI HUANG and WEI YANG \\ Department of Respiratory Medicine, Xiangya Hospital, Central South University; \\ Key Institute of National Clinical Research Center for Respiratory Disease of China, \\ Changsha, Hunan 410008, P.R. China
}

Received September 18, 2016; Accepted December 28, 2016

DOI: $10.3892 /$ or.2017.5751

\begin{abstract}
X-inactive specific transcript (XIST), one of the first found cancer-associated long non-coding RNAs (lncRNAs), is involved in the development and progression of many types of tumors. Aberrant expression of XIST has been observed in hepatocellular carcinoma, cervical, breast, ovarian and colorectal cancer. However, the exact effects and molecular mechanisms of XIST in lung cancer progression are still unknown to date. In the present study, we investigated the role of XIST in human lung cancer cell lines and clinical tumor samples in order to determine the function of this molecule. In our research, IncRNA-XIST was specifically upregulated in lung cancer cell lines and promoted lung cancer cell growth through targeting miR-140. Knockdown of XIST inhibited the proliferation and promoted cell apoptosis of human lung cancer cells and suppressed metastasis in vitro and in vivo. In addition, miR-140-dependent inhibitor of apoptosisstimulating protein of p53 (iASPP) regulation was required in XIST-induced lung cancer cell growth. These findings indicated that XIST may regulate the tumor growth and metastasis via miR-140-dependent iASPP regulation. Taken together, our data indicated that XIST may be an oncogenic lncRNA that promotes the proliferation and metastasis of lung cancer through the regulation of miR-140 and could be regarded as a therapeutic target in human lung cancer.
\end{abstract}

\section{Introduction}

Lung cancer is a tumor with a highly malignant phenotype characterized by rapid progression, late diagnosis, and a limited response to radiotherapy and chemotherapy. In the past 10 years, despite of the FDA approved therapeutic regimens and great improvements in medical care, we have observed

Correspondence to: Dr Yongjun Tang, Department of Respiratory Medicine, Xiangya Hospital, Central South University, 87 Xiangya Road, Hunan 410008, P.R. China

E-mail: tyj7736@163.com

Key words: XIST, miR-140, lung cancer, cell proliferation, cell apoptosis, iASPP no momentous effect on lung cancer patient survival $(1,2)$. Therefore, to identify biomarkers which may promote early diagnosis and allow personalized treatment strategies for patients at a high risk of lung cancer has become an urgent need (2).

MicroRNAs (miRNAs) are particularly important in almost all tumor developmental processes since they can be targets of genomic lesions, controlled by classic tumor signals, and they themselves present as a class of oncogenes or tumor suppressors $(3,4)$. Recently, a close association between miRNAs and lung cancer tumorigenesis has been suggested $(5,6)$. Multiple miRNAs, such as the let-7 family (7), miR-200 (8), miR-486 (9) and miR-146a (10) have been identified as tumor suppressors. Meanwhile, miR-31 (11), miR-212 (12) and miR-196a (13) were found to promote lung cancer carcinogenesis. miR-140 has been noted since it is involved in the development and progression of many types of cancers, including breast cancer (14), osteosarcoma (15), colon (16) and lung cancer (17). Yuan et al reported that miR-140 is significantly downregulated in non-small cell lung cancer (NSCLC) tissues and cell lines; and that miR-140 suppresses tumor growth and metastasis of NSCLC by targeting insulin-like growth factor 1 receptor (18). These findings suggest that miR-140 plays a tumor-suppressor role in these cancers. However, to the best of our knowledge, its role and the potential mechanisms in lung cancer remain unclear.

Human genome sequence data indicate that more than $90 \%$ of the DNA sequences actively transcribe, but only $2 \%$ encode protein; thus, the majority of the transcripts are referred to as non-coding RNAs (ncRNAs) (19,20). Small non-coding RNAs such as miRNAs have been studied extensively and their roles in gene regulation and cell function have been elucidated in numerous types of cancers (20). Recent studies have shown that long non-coding RNAs (lncRNAs) play important roles in both normal development and diseases including cancer (21). LncRNAs have emerged as new players in cancer research and several studies have shown that some lncRNAs function as oncogenes, tumor suppressor genes or both, depending on the circumstance (22).

The mechanisms by which lncRNAs exert their effect vary under different conditions; however, the interaction between lncRNAs and microRNAs plays a major role $(23,24)$. Zhu et al reported that the IncRNA H19/miR-675 axis 
represses prostate cancer metastasis by targeting transforming growth factor $\beta$-induced protein (TGFBI) (25). The interaction between miR-141 and lncRNA-H19 has been regarded as an important component in regulating cell proliferation and migration in gastric cancer (26).

In the present study, we report an interaction between $\mathrm{X}$-inactive specific transcript (XIST) and miR-140 which regulates lung cancer cell growth by directly targeting inhibitor of apoptosis-stimulating protein of p53 (iASPP). Our findings provide a novel understanding of the role of XIST and miR-140 in lung cancer metastasis and the mechanism involved.

\section{Materials and methods}

Tissue samples, cell lines and cell transfection. We collected 28 paired primary lung cancer and matched adjacent normal tissues. We obtained all samples from patients who underwent surgical resection at Xiangya Hospital of Central South University (Changsha, China). The tissues were snap-frozen in liquid nitrogen, and then stored at $-80^{\circ} \mathrm{C}$. The present study was approved by the Ethics Committee of Xiangya Hospital of Central South University. Informed consent statements were obtained from all the subjects included.

We purchased human lung cancer cell lines, A549 and H1299, from the American Type Culture Collection (ATCC; Manassas, VA, USA). They were cultured in RPMI-1640 medium (Invitrogen, Carlsbad, CA, USA) supplemented with $10 \%$ fetal bovine serum (Gibco, Carlsbad, CA, USA) at $37^{\circ} \mathrm{C}$ in a humidified atmosphere with $5 \% \mathrm{CO}_{2}$. The expression of miR-140 was regulated by transfection of miR-140 mimics or miR-140 inhibitor (GenePharma, Shanghai, China) using Lipofectamine 2000 (Invitrogen). A pcDNA3.1/iASPP was used to achieve overexpression of iASPP (GeneCopoecia, Guangzhou, China). Cells were plated into 6- or 96-well plates, transfected, incubated for 24 or $48 \mathrm{~h}$, and used for further assays or RNA/protein extraction.

RNA extraction and SYBR-Green quantitative PCR analysis. We extracted total RNA from cells using TRIzol reagent (Invitrogen), and detected mature miR-140 expression in cells using a Hairpin-it ${ }^{\mathrm{TM}}$ miRNAs qPCR kit (GenePharma). We used expression of RNU6B as an endogenous control. iASPP expression was measured using SYBR-Green qPCR assay (Takara, Dalian, China). Data were processed using the $2^{-\Delta \Delta \mathrm{Ct}}$ method.

MTT assay. A modified MTT assay was used to evaluate cell viability. After seeding $2 \times 10^{3}$ transfected cells/well into 96-well culture plates we assessed the viability of A549 and H1299 cells transfected with miR-140 or control at five time points (on day 1-5). In brief, quantification of mitochondrial dehydrogenase activity was achieved through the enzymatic conversion of [3-(4,5-dimethyldiazol-2-yl)-2,5-diphenyltetrazolium bromide (MTT); Sigma-Aldrich, St. Louis, MO, USA] to a colored formazan product. MTT $(10 \mu \mathrm{l}, 10 \mathrm{mg} / \mathrm{ml})$ was added to the cells, incubated for $4 \mathrm{~h}$, and the reaction was terminated by removal of the supernatant and addition of $100 \mu \mathrm{l}$ dimethyl sulfoxide (DMSO) to dissolve the formazan product. After $0.5 \mathrm{~h}$, the optical density (OD) of each well was measured at $570 \mathrm{~nm}$ using a plate reader (ELx808; Bio-Tek Instruments, Inc., Winooski, VT, USA).
BrdU incorporation assay. DNA synthesis in proliferating cells is determined by measuring 5-bromo-2'-deoxyuridine (BrdU) incorporation. BrdU assays were performed at 24 and $48 \mathrm{~h}$ after transfecting A549 and H1299 cells with miR-140 or the control vector. After seeding the infected cells in 96-well culture plates at a density of $2 \times 10^{3}$ cells/well, they were cultured for 24 or $48 \mathrm{~h}$, and incubated with a final concentration of $10 \mu \mathrm{M}$ BrdU (BD Pharmingen, San Diego, CA, USA) for 2-24 h. When the incubation period ended, we removed the medium, fixed the cells for $30 \mathrm{~min}$ at room temperature (RT), incubated them with peroxidase-coupled anti-BrdU antibody (Sigma-Aldrich) for $60 \mathrm{~min}$ at RT, washed them three times with phosphate-buffered saline (PBS), incubated the cells with peroxidase substrate (tetramethylbenzidine) for $30 \mathrm{~min}$, and measured the absorbance values at $450 \mathrm{~nm}$. Background BrdU immunofluorescence was determined in cells not exposed to BrdU, but stained with the BrdU antibody.

Apoptosis cell death detection by enzyme-linked immunosorbent assay (ELISA). To measure apoptosis in vitro, a DNA-fragmentation ELISA was used according to the manufacturer's instructions (Roche Diagnostics, Indianapolis, IN, USA).

Western blot analysis. The expression of iASPP and XIST in the lung cancer cells was detected by performing immunoblotting. We lysed cultured or transfected cells in RIPA buffer with $1 \%$ phenylmethanesulfonyl fluoride (PMSF), and loaded protein onto a SDS-PAGE minigel and transferred them onto polyvinylidene difluoride (PVDF) membranes. After being probed with 1:1,000 diluted rabbit polyclonal iASPP and XIST antibodies (Abcam, Cambridge, MA, USA) at $4^{\circ} \mathrm{C}$ overnight, the blots were subsequently incubated with the HRP-conjugated secondary antibody (1:5,000). ECL substrates were used to visualize signals (Millipore, Billerica, MA, USA). $\beta$-actin was used as an endogenous protein for normalization.

Luciferase reporter assay. A549 cells were seeded into a 24-well plate. After being cultured overnight, the cells were co-transfected with the wild-type and mutated iASPP 3' untranslated region (3' UTR) reporter plasmid, and pRL-TK plasmids, or transfected with miR-140 mimics and miR-140 inhibitor. Luciferase assays were performed $48 \mathrm{~h}$ after transfection using the Dual-Luciferase Reporter Assay System (Promega, Madison, WI, USA).

Statistical analysis. Data are expressed as the mean \pm SD of three independent experiments and were processed using SPSS 17.0 statistical software (SPSS, Inc., Chicago, IL, USA). Using Wilcoxon's paired test we compared the expression of miR-140 in lung cancer and the paired adjacent normal colonic tissues. The differences between groups were evaluated using the one-way analysis of variance (ANOVA). P-values of $<0.05$ were considered statistically significant.

\section{Results}

miR-140 negatively regulates $i A S P P$ expression in the A549 cell line. SYBR-Green quantitative PCR analysis was performed to quantify the expression of miR-140 in lung 


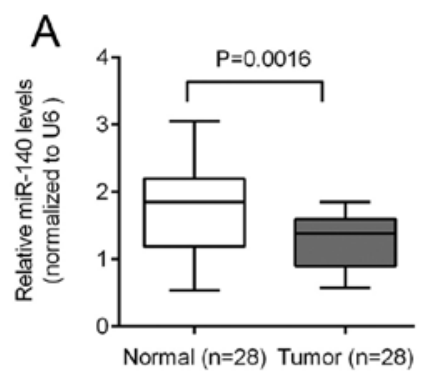

D

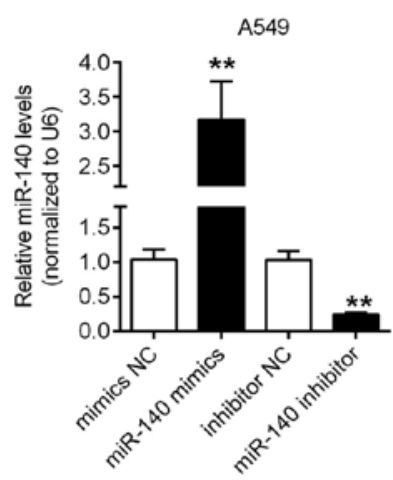

$\mathrm{F}$

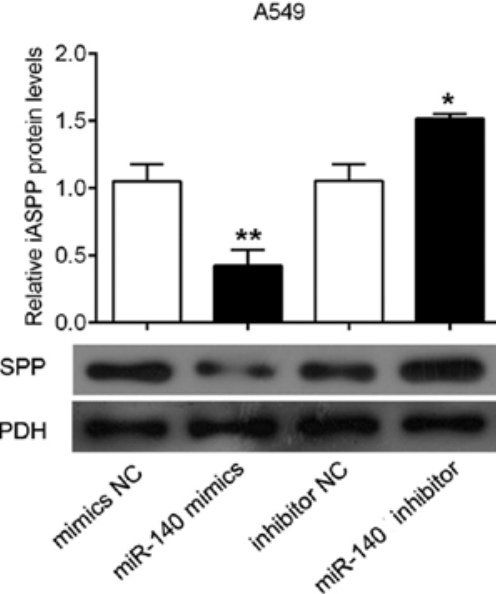

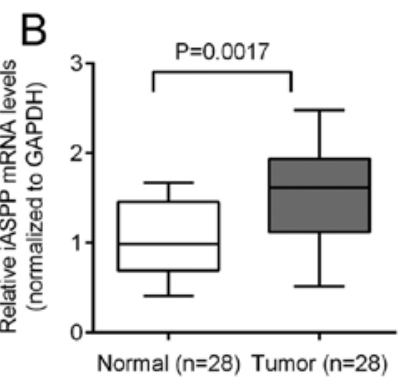

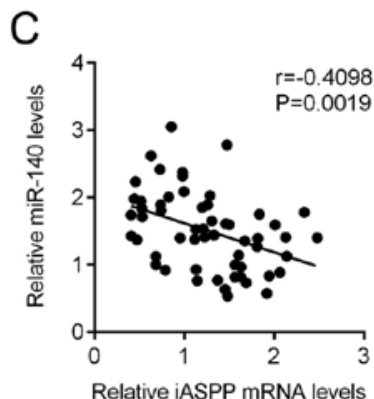

$\mathrm{E}$

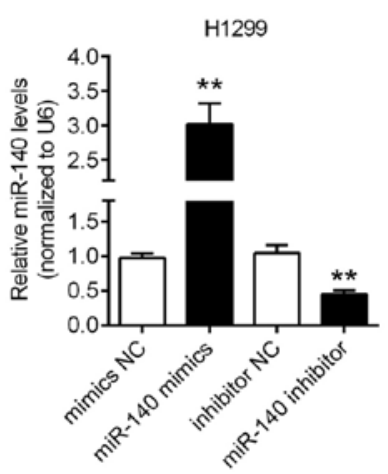

G

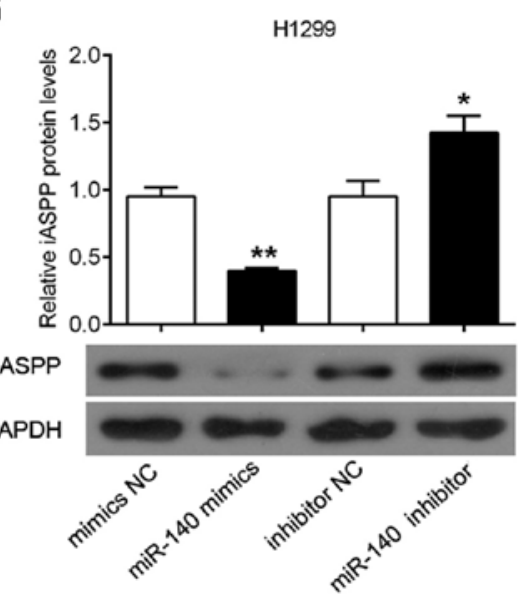

Figure 1. Inverse association of miR-140 and iASPP levels in lung tissues. (A) The miR-140 level was significantly downregulated in lung tissues compared with normal tissues. (B) iASPP level was markedly higher in lung tissues compared with normal tissues. (C) An inverse correlation between miR-140 and iASPP levels was observed. (D and E) miR-140 mimics were used to achieve miR-140 overexpression and miR-140 inhibitor was used to achieve suppression of miR-140 as confirmed by real-time qPCR in the A549 and H1299 cell lines. (F and G) miR-140 overexpression suppressed the iASPP protein expression, while miR-140 inhibition promoted the iASPP protein expression in both A549 and H1299 cell lines by western blotting and grey assay. The data are expressed as the mean $\pm \mathrm{SD}$ of three independent experiments. ${ }^{*} \mathrm{P}<0.05,{ }^{* *} \mathrm{P}<0.01$.

cancer tissues. In a large panel of 28 cases of primary lung cancer and the adjacent normal colonic tissues, the expression of miR-140 was significantly decreased in the lung cancer tissues, compared with that noted in the paired adjacent normal tissues (Fig. 1A). In contrast, the expression of iASPP was upregulated in the lung cancer tissues, compared with that noted in the paired adjacent normal tissues (Fig. 1B). An inverse correlation between the expression levels of miR-140 and iASPP was observed (Fig. 1C). To investigate the effect of miR-140 on iASPP expression, miR-140 mimics were used to achieve miR-140 overexpression, and miR-140 inhibitor was used to achieve miR-140 knockdown in the A549 and H1299 cell lines (Fig. 1D and E), and the expression efficiency was verified using real-time PCR. Next, the protein expression of iASPP was determined using western blotting in response to miR-140 overexpression or miR-140 inhibition. Results showed that miR-140 overexpression suppressed the iASPP protein expression, while miR-140 inhibition promoted the iASPP protein expression in both A549 and H1299 cell lines (Fig. 1F and G).

iASPP $m R N A$ is a direct target of $m i R-140$. It was predicted that miR-140 could bind to and target the iASPP 3' UTR using TargetScan, miRanda and miRWalk (27-29). A wt-iASPP 3' UTR luciferase reporter vector (wt-iASPP) was created to confirm this prediction, as well as a mut-iASPP $3^{\prime}$ UTR luciferase reporter vector (mut-iASPP) by sequentially mutating the predicted five base pair miR-140 binding site 
A

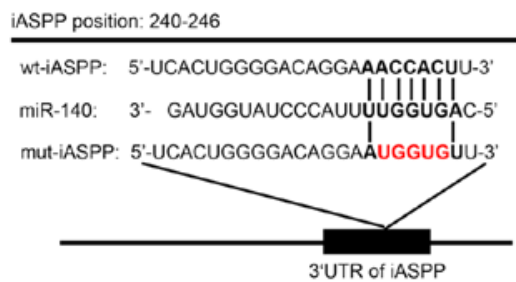

B

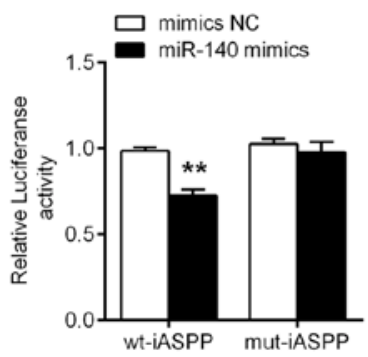

C

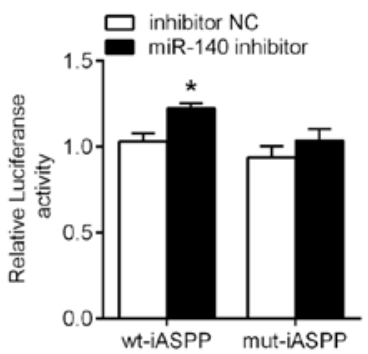

Figure 2. Interaction of miR-140 with the 3' UTR of iASPP. (A) A wt-iASPP and a corresponding mut-iASPP which contain a 5-bp mutation in the putative miR-140 binding site within its $3^{\prime}$ UTR was generated. (B) The wt-iASPP/mut-iASPP vectors and miR-140 NC/miR-140 mimics were co-transfected into A549 cells. The luciferase activity of the wt-iASPP reporter was significantly reduced by miR-140 mimic transfection, compared with the control groups; no significant reduction of the reporter activity was shown in cells co-transfected with miR-140 and the mut-iASPP reporter. (C) The wt-iASPP/mut-iASPP vectors and miR-140 NC/miR-140 inhibitor were co-transfected into A549 cells. The luciferase activity of the wt-iASPP reporter was increased by miR-140 inhibitor transfection. The data are expressed as the mean $\pm \mathrm{SD}$ of three independent experiments. ${ }^{*} \mathrm{P}<0.05,{ }^{* * *} \mathrm{P}<0.01$.

A

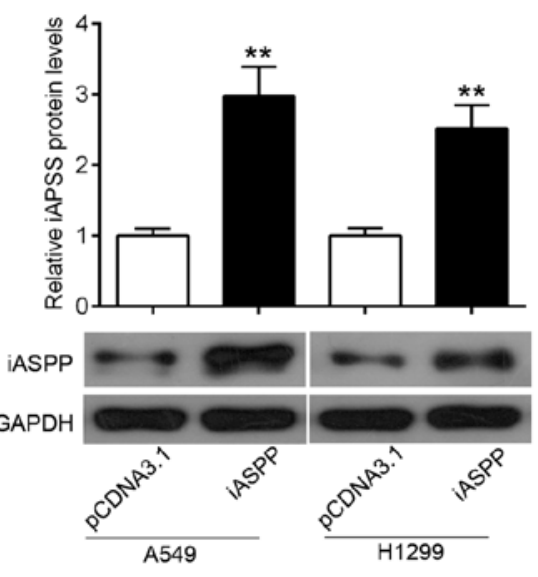

C

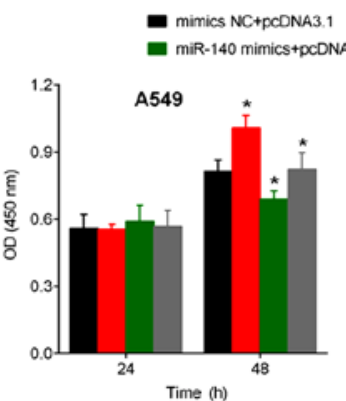

mimics NC+iASPP

miR-140 mimics+iASPP

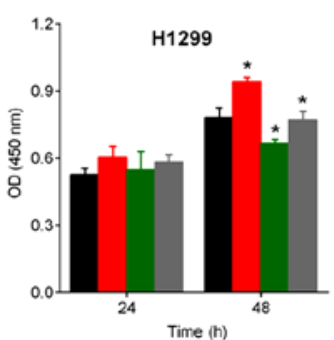

B

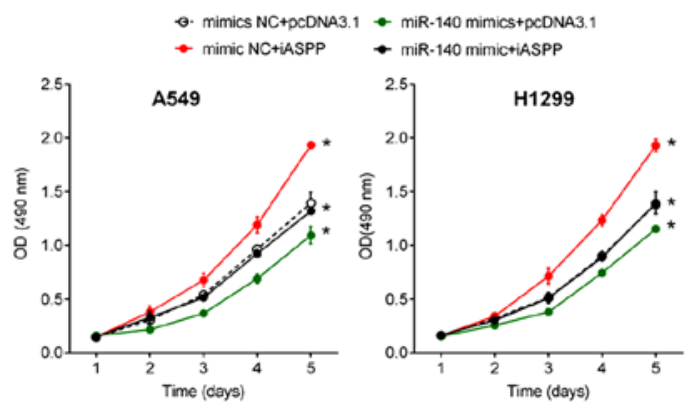

D

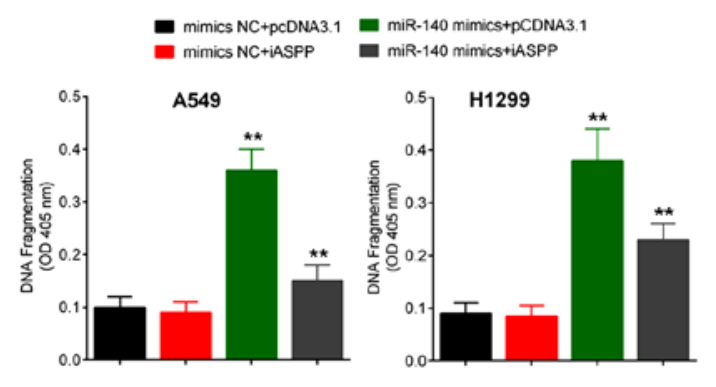

Figure 3. miR-140 inhibits the proliferation of the lung cancer cell lines through iASPP. (A) To investigate the functional roles of iASPP in lung cancer cells, we transfected A549 and H1299 cell lines with pcDNA3.1/iASPP to achieve iASPP overexpression. The expression level of iASPP protein was verified using western blotting. (B and C) pcDNA3.1/iASPP and miR-140 mimics/mimics NC were co-transfected into the A549 and H1299 cell lines. MTT and BrdU results showed that miR-140 mimic transfection significantly reduced the cell proliferation, while the forced expression of iASPP reversed the significant inhibition on cell proliferation by miR-140. (D) miR-140 overexpression notably promoted the DNA fragmentation of both A549 and H1299 cells, while iASPP transfection attenuated the promotive effect of miR-140 on DNA fragmentation of both A549 and H1299 cells. The data are showed as mean \pm SD of three independent experiments. ${ }^{*} \mathrm{P}<0.05,{ }^{* *} \mathrm{P}<0.01$.

in the $i A S P P$ 3' UTR (Fig. 2A). The wt-iASPP vector and miR-140 mimics or inhibitor were co-transfected into the A549 cell line. In the miR-140 mimic-transfected cells, the luciferase activity of the $i A S P P 3^{\prime}$ UTR luciferase reporter vector was significantly reduced while it was induced in the miR-140 inhibitor-transfected cells, compared to the inhibitor $\mathrm{NC}$ group (Fig. 2B and C). However, mutation of the putative the miR-140 binding site in the iASPP 3' UTR abolished miR- 140-mediated repression of $i A S P P 3^{\prime}$ UTR luciferase reporter activity (Fig. 2B and C).

miR-140 inhibits the proliferation of lung cancer cell line through iASPP. To investigate the functional roles of iASPP in lung cancer cells, we transfected A549 and H1299 cell lines with pcDNA3.1/iASPP to achieve iASPP overexpression (Fig. 3A). The expression level of iASPP protein was verified using 
A

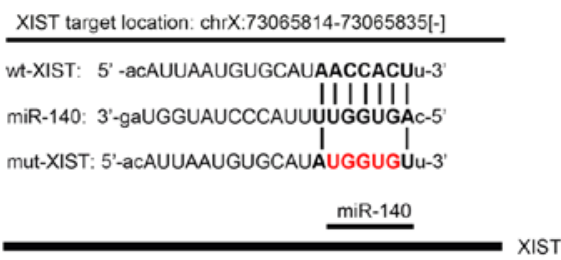

B

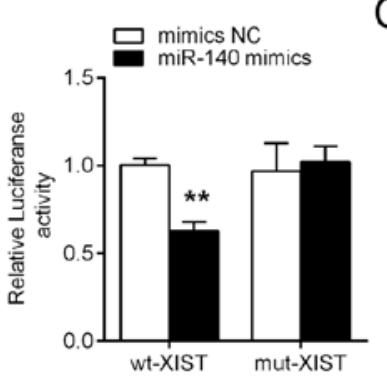

C

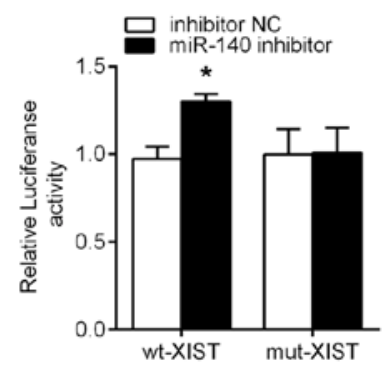

Figure 4. XIST is correlated with miR-140 by direct targeting. (A) A wt-XIST 3' UTR luciferase reporter vector (wt-XIST), as well as a mut-XIST3' UTR luciferase reporter vector (mut-XIST) by sequentially mutating predicted miR-140 binding sites in the XIST 3' UTR was generated. (B) The wt-XIST/mutXIST vectors and miR-140 NC/miR-140 mimics were co-transfected into A549 cells. The luciferase activity of the XIST luciferase reporter vector was significantly reduced in the miR-140 mimic-transfected cells, compared to the mimics NC groups. (C) The luciferase activity of the XIST luciferase reporter vector was markedly amplified in the miR-140 inhibitor-transfected cells compared to the inhibitor NC groups. The data are expressed as the mean \pm SD of three independent experiments. ${ }^{*} \mathrm{P}<0.05,{ }^{* *} \mathrm{P}<0.01$.

A

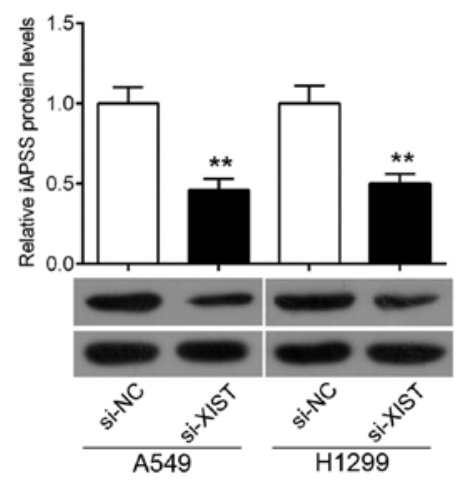

B

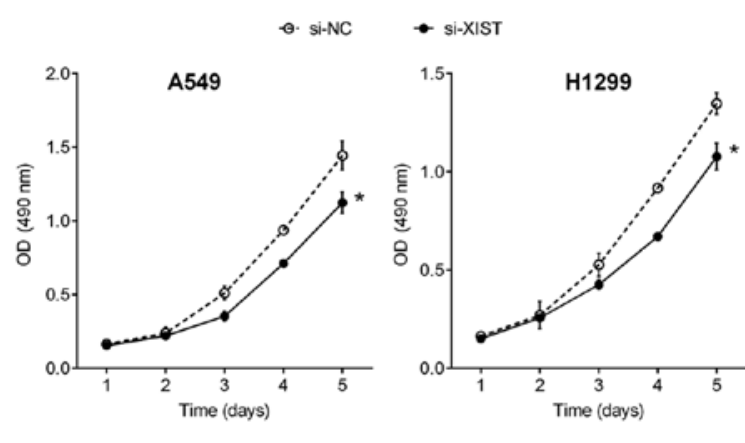

C

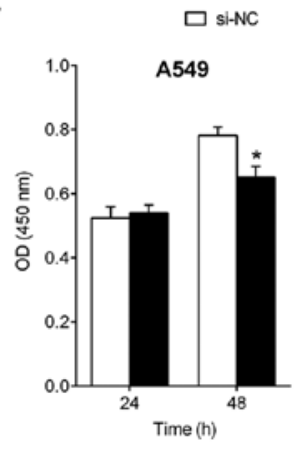

- si-XIST

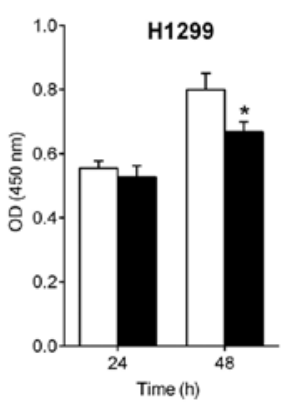

D

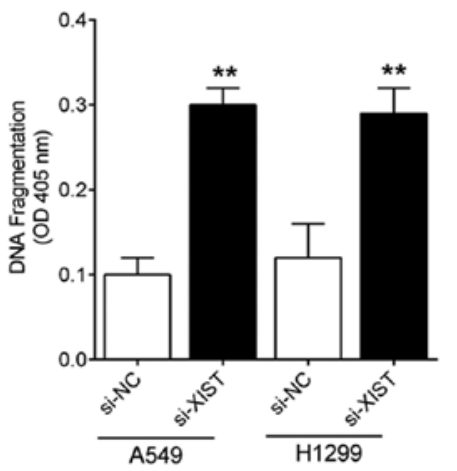

Figure 5. XIST promotes lung cancer cell growth and inhibits cell apoptosis. (A) XIST knockdown was achieved by si-XIST and the inhibitory efficiency was verified by real-time PCR. (B and C) MTT and BrdU assays revealed that knockdown of XIST significantly attenuated the proliferation of both A549 and H1299 cell lines over time, compared with the si-NC group. (D) DNA fragmentation of both A549 and H1299 cell lines was significantly promoted following knockdown of XIST. Taken together, these data revealed that lncRNA-XIST promoted lung cancer cell growth and inhibited cell apoptosis. The data are expressed as the mean $\pm \mathrm{SD}$ of three independent experiments. ${ }^{*} \mathrm{P}<0.05,{ }^{* *} \mathrm{P}<0.01$.

western blotting (Fig. 3A). Then, pcDNA3.1/iASPP and miR-140 mimics/mimics NC were co-transfected into the A549 and H1299 cell lines. MTT and BrdU results showed that miR-140 mimic transfection significantly reduced the cell proliferation, while the forced expression of iASPP reversed the significant inhibition on cell proliferation by miR-140 (Fig. 3B and C). Similarly, miR-140 overexpression notably promoted the DNA fragmentation of both A549 and H1299 cells, while iASPP transfection attenuated the promotive effect of miR-140 on DNA fragmentation of both A549 and H1299 cells (Fig. 3D).

XIST is correlated with miR-140 by direct targeting. According to previous studies, miR-140 plays a suppressive role in cancers (30). Previous studies have reported that XIST is associated with cancers by regulating miRNAs (31). To further investigate the mechanism by which miR-140 is regulated in lung cancer cell lines, we generated a wt-XIST 3' UTR luciferase 


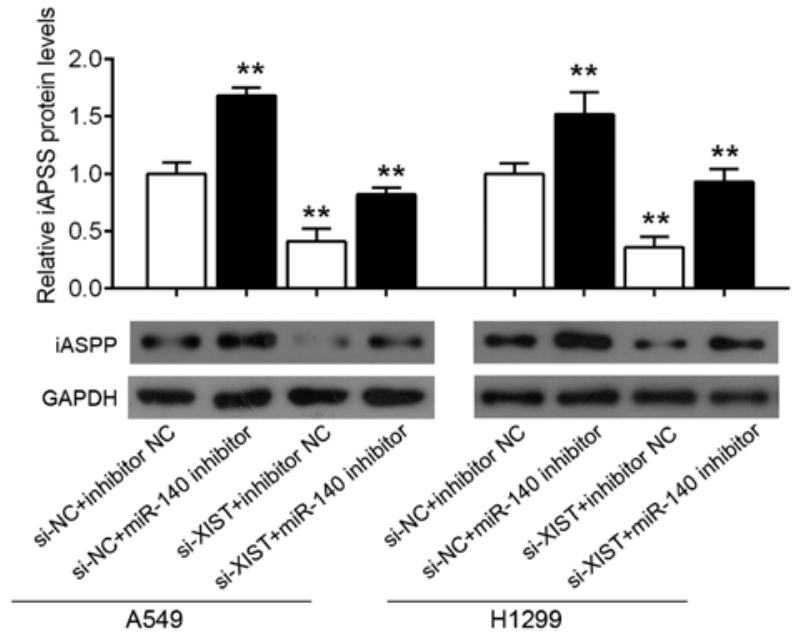

Figure 6. XIST promotes iASPP expression through miR-140. miR-140 knockdown significantly promoted iASPP protein expression, while XIST knockdown by si-XIST could partly restore this effect in both A549 and $\mathrm{H} 1299$ cell lines. The data are expressed as the mean \pm SD of three independent experiments. ${ }^{* *} \mathrm{P}<0.01$.

reporter vector (wt-XIST), as well as a mut-XIST3' UTR luciferase reporter vector (mut-XIST) by sequentially mutating predicted miR-140 binding sites in the XIST 3' UTR (Fig. 4A). We co-transfected the wt-XIST/mut-XIST vectors and miR-140 NC/miR-140 mimics into A549 cells. The luciferase activity of the XIST luciferase reporter vector was significantly reduced in the miR-140 mimic-transfected cells, compared to the mimics NC groups (Fig. 4B). Moreover, the luciferase activity of the XIST luciferase reporter vector was markedly amplified in the miR-140 inhibitor-transfected cells, compared to the inhibitor NC groups (Fig. 4C).

XIST promotes lung cancer cell growth and inhibits cell apoptosis. Next, we investigated the association of XIST expression with lung cancer cell proliferation. XIST knockdown was achieved by si-XIST and the inhibitory efficiency was verified by real-time PCR (Fig. 5A). Two lung cancer cell lines, A549 and H1299, were transfected with si-NC or si-XIST, and then the cell proliferation was determined by MTT and BrdU assays. MTT assays revealed that knockdown of XIST significantly attenuated the proliferation of both A549 and H1299 cell lines over time, compared with the si-NC group (Fig. 5B and C). Moreover, it was observed that DNA fragmentation of both A549 and H1299 cell lines was significantly promoted (Fig. 5D). Taken together, these data revealed that lncRNA-XIST promoted lung cancer cell growth and inhibited cell apoptosis.

XIST promotes iASPP expression through miR-140. Given that XIST inhibits miR-140 by direct binding, and that miR-140 inhibits iASPP by binding to its 3' UTR, we next investigated the association of XIST expression with iASPP protein expression in lung cancer cell lines. Results from western blotting showed that miR-140 knockdown significantly promoted iASPP protein expression, while XIST knockdown by si-XIST could partly restore this effect in both A549 and H1299 cell lines (Fig. 6A and B). Taken together, XIST promoted iASPP expression, most possibly through miR-140.

\section{Discussion}

Alterations in the presence of miRNAs are implicated in almost all fields of cancer biology, including cell growth, apoptosis, migration and/or invasion, and they function as either tumor suppressors or oncogenes (28). In the present study, we focused on miR-140 due to its potential suppressive function in human malignances. Song et al indicated that cell proliferation in both osteosarcoma and colon cancer cell lines was inhibited by overexpression of miR-140 (16). Recently, Yang et al reported that miR-140-5p was significantly decreased in HCC tissues and cell lines, and its overexpression suppressed tumor growth and metastasis by targeting transforming growth factor $\beta$ receptor 1 and fibroblast growth factor 9 (32). To date, however, the role of miR-140 in lung cancer carcinogenesis and the molecular mechanisms by which miR-140 exerts its functions remain unclear. In the present study, the real-time PCR results showed that miR-140 expression was significantly downregulated in lung cancer tissues. Overexpression of miR-140 significantly inhibited the proliferation and promoted the cell apoptosis of lung cancer cell. Accordingly, knockdown of miR-140 promoted cell proliferation and attenuated cell apoptosis. These results indicate that miR-140 could be a potential tumor-suppressor miRNA in lung cancer.

The impact of specific miRNAs on cancer biology depends on their downstream targets $(33,34)$. Different prediction algorithms were used to predict gene targets for miR-140, to elucidate the underlying mechanisms involved in the miR-140-induced inhibition of lung cancer growth and metastasis. The iASPP oncogene was identified as a critical downstream target of miR-140, due to its frequent overexpression in many malignancies and its function as an important regulator of cell proliferation, survival and metastasis (35-38). In the present study, miR-140 overexpression significantly downregulated the protein level of iASPP. Meanwhile, knockdown of miR-140 upregulated the expression of iASPP. A luciferase reporter assay validated the binding and repressive effects of miR-140 on the iASPP 3' UTR in A549 cells. Western blot analysis also confirmed that iASPP was downregulated by miR-140 upregulation, and upregulated by miR-140 downregulation. Moreover, the expression levels of miR-140 and iASPP in clinical lung cancer samples were significantly inversely correlated. To further confirm the role of iASPP in the A549 cell line, we revealed that knockdown of iASPP inhibited the proliferation and promoted the apoptosis of the A549 cell line. These data regarding the involvement of the miR-140/iASPP axis in lung cancer suggest that miR-140 may have potential as a therapeutic target.

To identify the correlation between miRNAs and lncRNAs and the underlying mechanisms provide potential targets for tumor treatment. Previous studies have mostly focused on the targets of miRNAs and the mechanisms by which miRNAs regulate the targets, thus as to influence tumor initiation and progression. For example, miRNAs including miR-21, miR-34a, miR-182 (39-41), have been reported to be involved in tumor growth and progression. However, few studies have focused on the regulation of miRNAs, particularly the co-regulation of miRNAs by non-coding RNAs. Emerging evidence has revealed that the mutual regulation between miRNAs and lncRNAs play major roles in tumor progression. 
In the present study, we revealed the interaction between XIST and miR-140 for the first time. The knockdown of XIST upregulated miR-140, while forced miR-140 overexpression inhibited the expression of XIST.

To detect the role of XIST in lung cancer cell growth regulation, siRNA was transfected into lung cancer cell lines to knock down XIST. As expected, iASPP was downregulated by XIST knockdown. In addition, the proliferation of lung cancer cell lines was reduced by iASPP inhibition. According to previous studies, iASPP is an oncogene and promotes cancer cell growth $(36,37,42)$.

To further validate the correlation of XIST, miR-140 and iASPP in lung cancer tissues, the expression levels of XIST, miR-140 and iASPP were determined. The data showed that the expression levels of XIST and iASPP were upregulated, while miR-140 expression was downregulated.

In summary, through targeting iASPP, lung cancer cell viability and proliferation were inhibited while cell apoptosis was promoted by miR-140. These effects were due to the regulation of miR-140 by XIST. The XIST/miR-140/iASPP axis identified in the present study may play a key role in regulating lung cancer cell proliferation and apoptosis, and may provide a potential therapeutic strategy for lung cancer.

\section{Acknowledgements}

The present study was supported by the National Key Scientific and Technology Support Program, and the Collaborative innovation of Clinical Research for Chronic Obstructive Pulmonary Sisease and Lung Cancer (no. 2013BAI09B09).

\section{References}

1. Neville A: Lung cancer. BMJ Clin Evid 2009: pii: 1504, 2009.

2. Chirieac LR and Dacic S: Targeted therapies in lung cancer. Surg Pathol Clin 3: 71-82, 2010.

3. Bartel DP: MicroRNAs: Genomics, biogenesis, mechanism, and function. Cell 116: 281-297, 2004.

4. Lan $\mathrm{H}, \mathrm{Lu} \mathrm{H}$, Wang $\mathrm{X}$ and Jin H: MicroRNAs as potential biomarkers in cancer: Opportunities and challenges. Biomed Res Int 2015: 125094, 2015.

5. Lai XL, Huang YH, Li YS, Li GN, Wang LP, Sun R, Ma YS, Feng SY, Chang ZY, Wang XH, et al: Differential expression profiling of microRNAs in para-carcinoma, carcinoma and relapse human pancreatic cancer. Clin Transl Oncol 17: 398-408, 2015.

6. Pavlakis E, Papaconstantinou I, Gazouli M, Theodosopoulos T, Karamanolis G, Genatas K and Ladas SD: MicroRNA gene polymorphisms in pancreatic cancer. Pancreatology 13: 273-278, 2013.

7. Li Y, VandenBoom TG II, Kong D, Wang Z, Ali S, Philip PA and Sarkar FH: Up-regulation of miR-200 and let-7 by natural agents leads to the reversal of epithelial-to-mesenchymal transition in gemcitabine-resistant pancreatic cancer cells. Cancer Res 69 6704-6712, 2009.

8. Yu J, Ohuchida K, Mizumoto K, Sato N, Kayashima T, Fujita H, Nakata K and Tanaka M: MicroRNA, hsa-miR-200c, is an independent prognostic factor in pancreatic cancer and its upregulation inhibits pancreatic cancer invasion but increases cell proliferation. Mol Cancer 9: 169, 2010.

9. Mees ST, Mardin WA, Sielker S, Willscher E, Senninger N, Schleicher C, Colombo-Benkmann M and Haier J: Involvement of $C D 40$ targeting miR-224 and miR-486 on the progression of pancreatic ductal adenocarcinomas. Ann Surg Oncol 16: 2339-2350, 2009.

10. Li Y, VandenBoom TG II, Wang Z, Kong D, Ali S, Philip PA and Sarkar FH: Up-regulation of miR-146a contributes to the inhibition of invasion of pancreatic cancer cells. Cancer Res 70 (Suppl 8): S5703, 2010.
11. Laurila EM, Sandström S, Rantanen LM, Autio R and Kallioniemi A: Both inhibition and enhanced expression of miR-31 lead to reduced migration and invasion of pancreatic cancer cells. Genes Chromosomes Cancer 51: 557-568, 2012.

12. Ma C, Nong K, Wu B, Dong B, Bai Y, Zhu H, Wang W, Huang X, Yuan Z and Ai K: miR-212 promotes pancreatic cancer cell growth and invasion by targeting the hedgehog signaling pathway receptor patched-1. J Exp Clin Cancer Res 33: 54, 2014.

13. Huang F, Tang J, Zhuang X, Zhuang Y, Cheng W, Chen W, Yao H and Zhang S: MiR-196a promotes pancreatic cancer progression by targeting nuclear factor kappa-B-inhibitor alpha. PLoS One 9: e87897, 2014.

14. Li Q, Yao Y,Eades G,Liu Z,Zhang Y and Zhou Q: Downregulation of miR-140 promotes cancer stem cell formation in basal-like early stage breast cancer. Oncogene 33: 2589-2600, 2014.

15. Hwang S, Park SK, Lee HY, Kim SW, Lee JS, Choi EK, You D, Kim CS and Suh N: miR-140-5p suppresses BMP2-mediated osteogenesis in undifferentiated human mesenchymal stem cells. FEBS Lett 588: 2957-2963, 2014

16. Song B, Wang Y, Xi Y, Kudo K, Bruheim S, Botchkina GI, Gavin E, Wan Y, Formentini A, Kornmann M, et al: Mechanism of chemoresistance mediated by miR-140 in human osteosarcoma and colon cancer cells. Oncogene 28: 4065-4074, 2009.

17. Kong XM, Zhang GH, Huo YK, Zhao XH, Cao DW, Guo SF Li AM and Zhang XR: MicroRNA-140-3p inhibits proliferation, migration and invasion of lung cancer cells by targeting ATP6AP2. Int J Clin Exp Pathol 8: 12845-12852, 2015.

18. Yuan Y, Shen Y, Xue L and Fan H: miR-140 suppresses tumor growth and metastasis of non-small cell lung cancer by targeting insulin-like growth factor 1 receptor. PLoS One 8: e73604, 2013.

19. Djebali S, Davis CA, Merkel A, Dobin A, Lassmann T, Mortazavi A, Tanzer A, Lagarde J, Lin W, Schlesinger F, et al: Landscape of transcription in human cells. Nature 489: 101-108, 2012.

20. Martens-Uzunova ES, Böttcher R, Croce CM, Jenster G, Visakorpi T and Calin GA: Long noncoding RNA in prostate, bladder, and kidney cancer. Eur Urol 65: 1140-1151, 2014.

21. Ponting CP, Oliver PL and Reik W: Evolution and functions of long noncoding RNAs. Cell 136: 629-641, 2009.

22. Zhou S, Wang J and Zhang Z: An emerging understanding of long noncoding RNAs in kidney cancer. J Cancer Res Clin Oncol 140: 1989-1995, 2014.

23. Paraskevopoulou MD and Hatzigeorgiou AG: Analyzing miRNA-lncRNA interactions. Methods Mol Biol 1402: 271-286, 2016.

24. Wu Z, Liu X, Liu L, Deng H, Zhang J, Xu Q, Cen B and Ji A: Regulation of lncRNA expression. Cell Mol Biol Lett 19: 561-575, 2014.

25. Zhu M, Chen Q, Liu X, Sun Q, Zhao X, Deng R, Wang Y, Huang J, $\mathrm{Xu}$ M, Yan J, et al: lncRNA H19/miR-675 axis represses prostate cancer metastasis by targeting TGFBI. FEBS J 281: 3766-3775, 2014.

26. Zhou X, Ye F, Yin C, Zhuang Y, Yue G and Zhang G: The interaction between miR-141 and lncRNA-H19 in regulating cell proliferation and migration in gastric cancer. Cell Physiol Biochem 36: 1440-1452, 2015.

27. Esquela-Kerscher A and Slack FJ: Oncomirs - microRNAs with a role in cancer. Nat Rev Cancer 6: 259-269, 2006.

28. Hwang HW and Mendell JT: MicroRNAs in cell proliferation, cell death, and tumorigenesis. Br J Cancer 96 (Suppl): R40-R44, 2007.

29. Lewis BP, Burge CB and Bartel DP: Conserved seed pairing, often flanked by adenosines, indicates that thousands of human genes are microRNA targets. Cell 120: 15-20, 2005.

30. Green D, Dalmay T and Fraser WD: Role of $m i R-140$ in embryonic bone development and cancer. Clin Sci 129: 863-873, 2015.

31. Yao Y, Ma J, Xue Y, Wang P, Li Z, Liu J, Chen L, Xi Z, Teng H, Wang Z, et al: Knockdown of long non-coding RNA XIST exerts tumor-suppressive functions in human glioblastoma stem cells by up-regulating miR-152. Cancer Lett 359: 75-86, 2015.

32. Yang H, Fang F, Chang R and Yang L: MicroRNA-140-5p suppresses tumor growth and metastasis by targeting transforming growth factor $\beta$ receptor 1 and fibroblast growth factor 9 in hepatocellular carcinoma. Hepatology 58: 205-217, 2013.

33. Profumo V, Doldi V, Gandellini P and Zaffaroni N: Targeting microRNAs to withstand cancer metastasis. Methods Mol Biol 1218: 415-437, 2015. 
34. Chen J, Sun D, Chu H, Gong Z, Zhang C, Gong B, Li Y, Li N and Jiang L: Screening of differential microRNA expression in gastric signet ring cell carcinoma and gastric adenocarcinoma and target gene prediction. Oncol Rep 33: 2963-2971, 2015.

35. Chen J, Xie F, Zhang L and Jiang WG: iASPP is over-expressed in human non-small cell lung cancer and regulates the proliferation of lung cancer cells through a p53 associated pathway. BMC Cancer 10: 694, 2010.

36. Gillotin S: iASPP, a potential drug target in cancer therapy. Leuk Res 33: 1175-1177, 2009.

37. Liu T, Li L, Yang W, Jia H, Xu M, Bi J, Li Z, Liu X, Li Z, Jing H, et al: iASPP is important for bladder cancer cell proliferation. Oncol Res 19: 125-130, 2011

38. Jia Y, Peng L, Rao Q, Xing H, Huai L, Yu P, Chen Y, Wang C, Wang M, Mi Y, et al: Oncogene iASPP enhances self-renewal of hematopoietic stem cells and facilitates their resistance to chemotherapy and irradiation. FASEB J 28: 2816-2827, 2014.
39. Luan S, Sun L and Huang F: MicroRNA-34a: A novel tumor suppressor in p53-mutant glioma cell line U251. Arch Med Res 41: 67-74, 2010.

40. Li S, Liang Z, Xu L and Zou F: MicroRNA-21: A ubiquitously expressed pro-survival factor in cancer and other diseases. Mol Cell Biochem 360: 147-158, 2012.

41. Bai AH, Milde T, Remke M, Rolli CG, Hielscher T, Cho YJ, Kool M, Northcott PA, Jugold M,Bazhin AV, et al: MicroRNA-182 promotes leptomeningeal spread of non-sonic hedgehog-medulloblastoma. Acta Neuropathol 123: 529-538, 2012.

42. Li Y, Ahmad A and Sarkar FH: ASPP and iASPP: Implication in cancer development and progression. Cell Mol Biol 61: 2-8, 2015. 